\title{
An aggressive fibromatosis in the palm of a female child: A rare case
}

\author{
Arun Ranjan Napit ${ }^{1}$, Shraddha Panchal ${ }^{2}$, Dhaval Panchal ${ }^{3}$ \\ From ${ }^{1}$ Medical Officer, Department of General OPD, Valley Health and Research Center, Bhaktapur, Nepal, ${ }^{2}$ Medical Officer, Department of Emergency, \\ Krishna Hospital, Ahmedabad, ${ }^{3}$ Surgeon, Department of Surgery, Krishna Hospital, Ahmedabad, Gujarat, India
}

\begin{abstract}
Aggressive fibromatosis is the locally aggressive benign tumor of mesenchymal origin. It can be found in any part of the body. However, abdomen is the most common site of this lesion. It has a predilection to females between 15 and 60 years. We report the case of a 16-month-old female child with an aggressive fibromatosis in her right palm. The location and age of presentation make this a rare case. She was diagnosed by histopathological examination and the mass was excised. The patient was advised for follow-up examination due to the high degree of recurrence of this tumor.
\end{abstract}

Key words: Aggressive fibromatosis, Collagenous stroma, Trucut biopsy

A ggressive fibromatosis is a benign tumor of mesenchymal origin. It is locally aggressive but does not metastasize [1]. It is also called a desmoid tumor. The age of presentation of this condition is between 15 and 60 years. In the general population, aggressive fibromatosis has an incidence of 2-4 per million worldwide, with slightly predominant in females. Due to its origin from the connective tissue, aggressive fibromatosis can arise from any part of the body. However, it is often discovered in the abdomen, followed by the shoulder, upper arms, and thigh [2]. It is also found to be associated with trauma, pregnancy, and Gardner syndrome [3]. Approximately 5-10\% of the cases of fibromatosis run in the family [2]. Due to their tendency to invade nearby tissue and tendency to recur, the World Health Organization has classified this tumor as an "intermediate locally aggressive tumor" [4].

\section{CASE REPORT}

A 16-month-old female child presented to the outpatient department with complaints of the right palm swelling for 1 year. The swelling was insidious in onset that gradually increased in size, ultimately covering the whole palm area. The patient had no complaints of fever, pain, and trauma. There were no significant past and family histories.

On arrival, the patient vitals were normal. On examination, there was a firm swelling covering the entire palmar surface of the right hand (Fig. 1a and b). No local inflammatory signs were found.

\section{Access this article online}

Received - 15 September 2021

Initial Review - 01 October 2021

Accepted - 01 November 2021

DOI: $10.32677 /$ ijer.v7i11.3121
Computed tomography (CT) scan of the right palm was ordered that showed a multilobulated hypodense soft-tissue lesion of approximately $22 \times 46 \times 54 \mathrm{~mm}$ size along the palmer aspect of the right hand (Fig. 2a and b). There was encasement of muscles and tendons along the palmar aspect with loss of fat planes, representing probably as a fibrosing tumor.

A trucut biopsy of the lesion on the right palm was done and was sectioned and stained with hematoxylin and eosin stain. The microscopy showed hypocellular fibrous tissue revealing spindle or stellate cells widely separated by abundant collagenous stroma. Few adnexal structures and fragmented epidermis were also noted (Fig. 3a and b). There was no mitosis, nuclear atypia, inclusion, hyaline deposit, or vasculitis favoring the histological diagnosis of fibromatosis (Fig. 3c and d).

The patient was admitted to daycare. After admission, general examinations including vitals and necessary blood investigations (Comprehensive Metabolic Panel, Human Immunodeficiency Virus, Hepatitis B surface antigen, and Hepatitis C virus) were done and found normal. She underwent the excision of the mass under general anesthesia. She tolerated the procedure well. Volar approach (modified Henry extension) was done and the lesion was marginally excised. Ulnar nerve and median nerves were protected and were prophylactically decompressed in the Guyon canal. Hypothenar muscles (abductor digiti minimi, flexor digiti minimi, and opponens digiti mini) were completely encased in mass, so they were removed with the tumor. The remaining thenar muscles, flexor digitorum superficialis, and flexor digitorum profundus were protected.

Postoperatively, she was shifted to the daycare and managed with cefuroxime $250 \mathrm{mg} \mathrm{BD}$, ibuprofen $5 \mathrm{ml} \mathrm{BD}$, pantoprazole $20 \mathrm{mg}$ OD, intravenous fluids, and other supportive measures.

Correspondence to: Dr. Arun Ranjan Napit, Byasi-02, Bhaktapur, Nepal. E-mail: arunranjannapit@gmail.com

(C) 2021 Creative Commons Attribution-NonCommercial 4.0 International License (CC BY-NC-ND 4.0). 


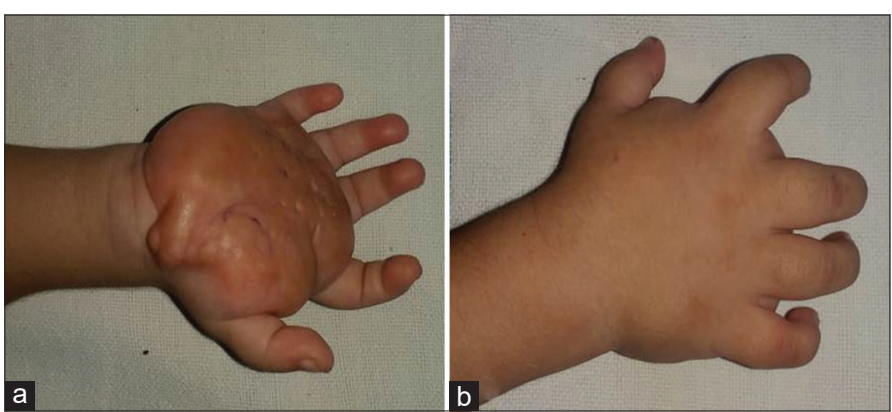

Figure 1: Clinical photographs of the patient showing swelling of the right hand (a) ventrally; (b) dorsally

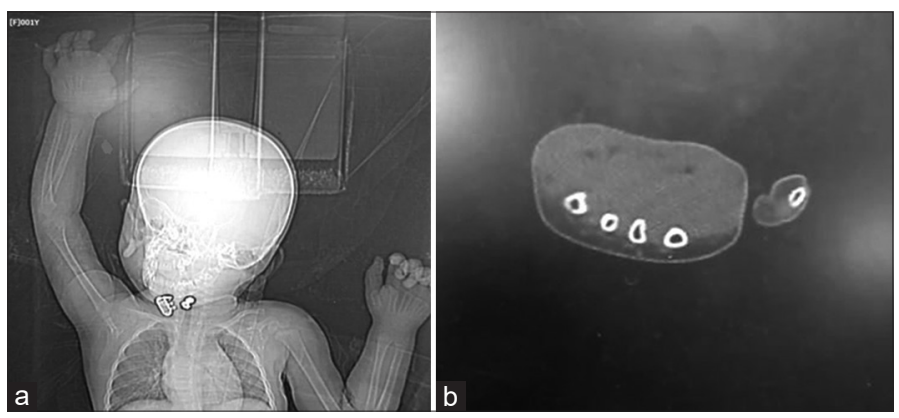

Figure 2: (a and b) Computed tomography images showing mass in the right hand

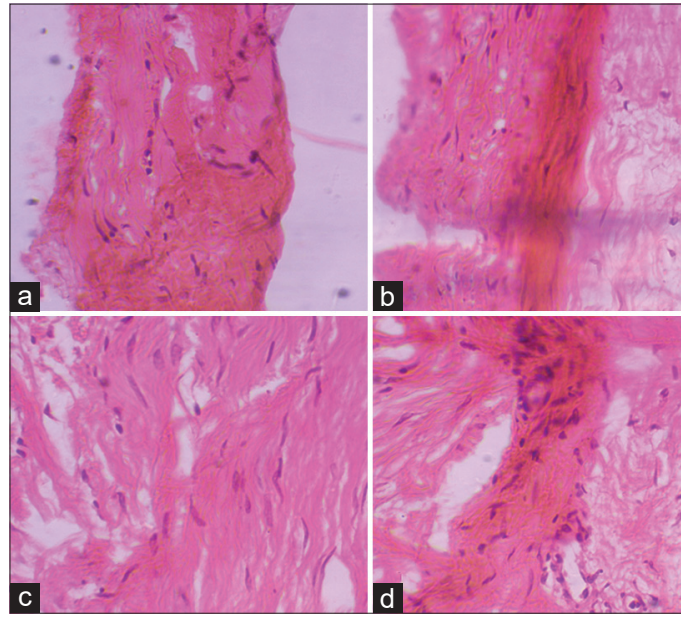

Figure 3: (a-d) The H\&E stained histological images showing proliferation of fibroblast in the background of collagenous stroma suggestive of aggressive fibromatosis

Nil by mouth was cleared and the patient tolerated the oral diet well. She mobilized well, with no fresh complaints. Hence, given her stable vitals, she was discharged after explaining further care and follow-up advice. Although she was advised to come for follow-up at 1 month, 3 months, 6 months, and follow-ups have to be maintained for at least 2 years for recurrence; the patient came for follow-up visits for only 3 months. She was able to do her activity of daily living. She had complete atrophy of hypothenar eminence but was able to do remaining hand function.

\section{DISCUSSION}

Aggressive fibromatosis, also called a desmoid tumor, is a locally aggressive tumor that originates from fibroblast cells. These cells are a part of the connective tissue. Since connective tissues are present everywhere in the body, aggressive fibromatosis can occur in any part of the body. However, the abdomen is the most common site of presentation [5]. Aggressive fibromatosis is not encapsulated with a poorly defined margin. Hence, it has a high tendency to invade local tissue and recur after treatment [6]. Local invasion to the neurovascular structure can cause pain and loss of function [4].

Although the exact etiopathogenesis of aggressive fibromatosis is still unknown, it is considered to be sporadic or hereditary. Trauma and surgical scar sites have a higher tendency to develop this condition. Similarly, pregnancy and oral contraceptive use is also associated with the development of aggressive fibromatosis [4]. Furthermore, studies have shown that this condition has more predilections toward females [5]. Association of the desmoid tumor with hereditary conditions such as Gardner syndrome and familial adenomatous polyposis (FAP) supports the hereditary nature of this disease [3]. Approximately $85 \%$ of sporadic cases show a mutation in the beta-catenin pathway. It is known that $45 \mathrm{~F}$ mutation in the beta-catenin pathway is associated with an increased lesion recurrence rate. Similarly, desmoid tumor associated with FAP shows a mutation in the APC gene. Moreover, this desmoid tumor has a higher tendency to grow at the surgical scar site [6].

Ultrasound, CT scan, and magnetic resonance imaging (MRI) are used for the imaging of aggressive fibromatosis for investigation and follow-up. They help define the origin and extent of local invasion of the lesion and guide the surgery plan [6]. Although MRI is more sensitive, CT scan is commonly used for imaging this tumor [4]. It presents as soft-tissue mass with poor margin and infiltration of the surrounding tissue. Metastasis is always absent here [3]. The diagnosis of aggressive fibromatosis is made by histological findings. It presents as the proliferation of spindle-shaped fibroblast cells, massive collagenous stroma, and blood vessels without nuclear atypia and hyperchromasia. It also stains positive for beta-catenin, vimentin, cyclooxygenase-2, tyrosine kinase, and estrogen receptor beta [6].

Surgery is the mainstream treatment for aggressive fibromatosis. However, it is associated with recurrence and a high degree of morbidity and mortality [7]. The limitation of surgery can be overcome by radiotherapy alone or radiotherapy along with surgery as a treatment modality [8]. Other treatment options for aggressive fibromatosis are chemotherapy (methotrexate, tyrosine kinase inhibitor, and vinblastine), hormonal therapy (tamoxifen and raloxifene), non-steroidal anti-inflammatory drugs, high-intensity focused ultrasound treatment, and ablation technique (radiofrequency ablation) [1].

Recurrence is common in aggressive fibromatosis. Although age, size, and site of the tumor have no association with recurrence, a positive margin has a high chance of recurrence. Hence, a follow-up for at least 2 years with a wait and watch policy followed by adjuvant radiotherapy is offered in case of recurrence [9]. 


\section{CONCLUSION}

Aggressive fibromatosis in the palm of a 16-month female child is a rare case in terms of the lesion site and age of presentation. Due to the lack of capsule and its nature of the aggressive local invasion, the patient has to be followed up for the possibility of recurrence.

\section{REFERENCES}

1. Fibromatosis-an Overview. ScienceDirect Topics; 2021. Available from: https://www.sciencedirect.com/topics/pharmacology-toxicology-andpharmaceutical-science/fibromatosis [Last accessed on $2021 \mathrm{Sep} 02]$.

2. Desmoid Tumor-National Cancer Institute; 2019. Available from: https:// www.cancer.gov/pediatric-adult-rare-tumor/rare-tumors/rare-soft-tissuetumors/desmoid-tumor [Last accessed on $2021 \mathrm{Sep}$ 02].

3. Weerakkody Y. Aggressive Fibromatosis. Radiology Reference Article, Radiopaedia; 2021. Available from: https:/www.radiopaedia.org/articles/ aggressive-fibromatosis [Last accessed on 2021 Oct 22].

4. Moorthy GD, Amini B, Nikolaidis P, Assing M, Vikram R. Current update on desmoid fibromatosis. J Comput Assist Tomogr 2019;43:29-38.
5. Peña S, Brickman T, StHilaire H, Jeyakumar A. Aggressive fibromatosis of the head and neck in the pediatric population. Int J Pediatr Otorhinolaryngol 2014;78:1-4.

6. Master SR, Mangla A, Puckett Y, Shah C. Desmoid tumor. In: Stat Pearls. Treasure Island, FL: Stat Pearls Publishing; 2021.

7. Zhang Z, Shi J, Yang T, Liu T, Zhang K. Management of aggressive fibromatosis. Oncol Lett 2021;21:43.

8. Kasper B, Baumgarten C, Garcia J, Bonvalot S, Haas R, Haller F, et al. An update on the management of sporadic desmoid-type fibromatosis: A European consensus initiative between sarcoma patients EuroNet (SPAEN) and European organization for research and treatment of cancer (EORTC)/soft tissue and bone sarcoma group (STBSG). Ann Oncol 2017;28:2399-408.

9. Ramamurthy R, Arumugam B, Ramanandham B. Recurrence patterns and management options in aggressive fibromatosis. Indian J Surg Oncol 2012;3:222-7.

Funding: None; Conflicts of Interest: None Stated.

How to cite this article: Napit AR, Panchal S, Panchal D. An aggressive fibromatosis in the palm of a female child: A rare case. Indian J Case Reports. 2021;7(11):482-484. 